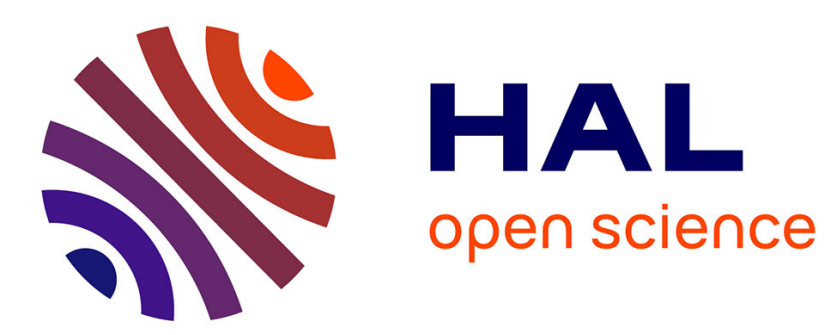

\title{
Observer design for networked control systems with FlexRay
}

Wei Wang, Dragan Nesic, Romain Postoyan

\section{To cite this version:}

Wei Wang, Dragan Nesic, Romain Postoyan. Observer design for networked control systems with FlexRay. Automatica, 2017, 82, pp.42-48. 10.1016/j.automatica.2017.03.038 . hal-01521591

\section{HAL Id: hal-01521591 \\ https://hal.science/hal-01521591}

Submitted on 12 May 2017

HAL is a multi-disciplinary open access archive for the deposit and dissemination of scientific research documents, whether they are published or not. The documents may come from teaching and research institutions in France or abroad, or from public or private research centers.
L'archive ouverte pluridisciplinaire HAL, est destinée au dépôt et à la diffusion de documents scientifiques de niveau recherche, publiés ou non, émanant des établissements d'enseignement et de recherche français ou étrangers, des laboratoires publics ou privés. 


\title{
Observer design for networked control systems with FlexRay *
}

\author{
Wei Wang ${ }^{\mathrm{a}}$, Dragan Nešić ${ }^{\mathrm{a}}$, Romain Postoyan ${ }^{\mathrm{b}}$ \\ ${ }^{a}$ Department of Electrical and Electronic Engineering, the University of Melbourne, Parkville, 3010, Victoria, Australia \\ ${ }^{\mathrm{b}}$ Université de Lorraine, CRAN, UMR 7039 and CNRS, CRAN, UMR 7039, France
}

\begin{abstract}
We design state observers for nonlinear networked control systems (NCS) implemented over FlexRay. FlexRay is a communication protocol used in the automotive industry, which has the feature to switch between two scheduling rules during its communication cycles. These switches induce technical difficulties when modeling, designing and analysing observers for such systems compared to standard NCS. We present a solution based on the emulation approach. Given an observer in the absence of communication constraints, we implement it over the network and we provide sufficient conditions on the latter, to preserve the stability property of the observer. In particular, we provide explicit bounds on the maximal allowable transmission intervals, which adapt to the lengths of the segment associated to each scheduling rule. We assume that the plant dynamics and measurements are affected by noise and we guarantee an input-to-state stability property for the corresponding estimation error system. The overall system is modeled as a hybrid system and the analysis relies on the use of a novel hybrid Lyapunov function.
\end{abstract}

\section{Introduction}

Networked control systems (NCS) attract great attention due to the features they offer in terms of ease of installation and flexibility. NCS are characterized by the use of a shared serial communication channel to connect spatially distributed sensors and actuators with the control unit. While the usage of a network offers great advantages over traditional point-to-point set-ups, it also introduces communication constraints in terms of limited sampling, scheduling, delay, packet loss, quantization, which may have a severe impact on the desired requirements. In this paper, we focus on FlexRay networks and we concentrate on the effect of sampling and scheduling. FlexRay is a protocol developed by BMW, Daimler-Chrysler, Philips and Freescale in 2000 to provide appropriate communications for implementing $\mathrm{X}$ by-wire technology in automotive control (Consortium 2005, Schmidt \& Schmidt 2009). It works with communication cycles, that alternate between a static and a dynamic segment during which a specific scheduling rule

\footnotetext{
* Supported by the Australian Research Council under the Discovery Project (DP170104099), and the ANR under the grant COMPACS (ANR-13- BS03-0004-02).

Email addresses: wweiqust@gmail.com (Wei Wang), dnesic@unimelb.edu.au (Dragan Nešić), romain.postoyan Quniv-lorraine.fr (Romain Postoyan).
}

is used (Consortium 2005); we thus have to deal with a switched protocol. Our objective is to design observers for uncertain nonlinear NCS with FlexRay. This study is motivated by the fact that this communication protocol is increasingly used in the automotive industry and estimation methods for such systems are currently missing in the literature; only stabilization results are available to the best of our knowledge, see e.g. (Naghshtabrizi \& Hespanha 2009, Wang et al. 2015).

We consider the scenario where the plant input and the measurements are sent to the observer via the network. Actuators and sensors are grouped into nodes, and only one of these transmit its data to the observer at each transmission instant. The latter therefore only has access to partial and sampled information of the plant input and output. The solution we propose is based on emulation. The idea is to first synthesize the observer while ignoring the communication constraints. At this stage, any of the continuous-time observer design techniques available in the literature can be applied. Then, the observer is implemented over the network and conditions on the latter are derived to preserve the desired error convergence properties. Similar results exist for NCS with nonswitched protocols see (Postoyan \& Nešić 2012, Postoyan et al. 2014), however none of these apply to our problem because of the switches between the two scheduling rules exhibited by FlexRay. Indeed, these switches re- 
quire a new model, appropriate assumptions on the observer and the network, and a new stability analysis. In addition, we consider plants with input, perturbed dynamics and noisy measurements, which is more general and induces additional technical difficulties compared with (Postoyan \& Nešić 2012, Postoyan et al. 2014).

We model the overall system as a hybrid system in the formalism of (Goebel et al. 2012), for which a jump either describes a segment switch or a transmission. We assume that the transmissions during the static and the dynamic segments are governed by possibly (distinct) input-tostate stable (ISS) protocols, which include round-robin (RR) and maximum-error-first try-once-discard (TOD) (Walsh \& Ye 2001), as particular cases. The concept of ISS protocol was introduced in (Tabbara \& Nešić 2008) and appears to be very useful for NCS subject to measurement noise. We provide explicit bounds on the maximum allowable transmission intervals (MATI) for each segment and we guarantee an input-to-state stability property for the estimation error system. The analysis relies on a novel hybrid Lyapunov function.

Our contributions are threefold. First, we present an observer design strategy for NCS with FlexRay for the first time to the best of our knowledge. Second, the MATI bounds we propose are much simpler to compute than those derived in (Wang et al. 2015), where the corresponding stabilization problem is addressed. This is due to the novel hybrid Lyapunov function we construct. Third, our results extend the works in (Postoyan \& Nešić 2012, Postoyan et al. 2014) to perturbed systems with control inputs and noisy measurements in the particular case where there is a single segment.

\section{Problem Statement}

Consider the nonlinear plant

$$
\dot{x}_{p}=\mathbf{f}_{p}\left(x_{p}, u, w\right) \quad y_{p}=\mathbf{g}\left(x_{p}\right)+v,
$$

where $x_{p} \in \mathbb{R}^{n_{x}}$ is the state, $u \in \mathbb{R}^{n_{u}}$ is the control input, $w \in \mathbb{R}^{n_{w}}$ is the external disturbance, $y_{p} \in \mathbb{R}^{n_{y}}$ is the plant output affected by the noise $v \in \mathbb{R}^{n_{y}}$. The functions $u(\cdot)$ and $v(\cdot)$ are assumed to be Lebesgue measurable and differentiable. Moreover, these functions and their time-derivatives are assumed to have a finite $\mathcal{L}_{\infty}$ norm. We assume that we know an observer of the form

$$
\dot{x}_{o}=\mathbf{f}_{o}\left(x_{o}, u, y_{p}-y_{o}\right) \quad y_{o}=\mathbf{g}\left(x_{o}\right),
$$

where $x_{o} \in \mathbb{R}^{n_{x}}$ is the estimate of the state $x_{p}$ and $y_{o} \in$ $\mathbb{R}^{n_{y}}$ is the output of the observer.

We consider the scenario where the plant and the observer communicate over a network and FlexRay is used to schedule transmissions, see Figure 1. In particular, the control signal $u$ is directly available to the plant and transmitted to the estimation unit via the network to the observer as $\hat{u}$. We assume that the signal $u$ and the

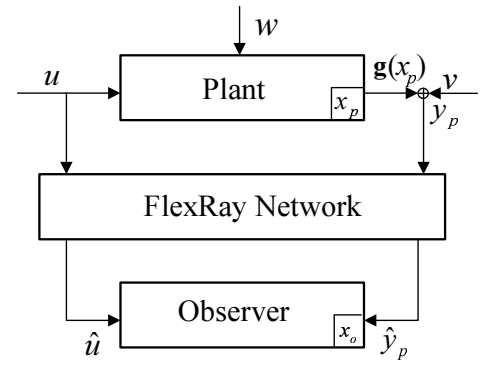

Fig. 1. Block diagram of the setup

noisy measurements $y_{p}$ are transmitted through $\ell \in \mathbb{Z}_{>0}$ nodes, where a node corresponds to a group of co-located sensors or/and actuators, and the latter have access to the measurements of some components of $\left(y_{p}, u\right)$. Because of the network, the observer has no longer access to $y_{p}$ and $u$, but to their networked versions $\hat{y}_{p}$ and $\hat{u}$. At each transmission instant $t_{i}, i \in \mathbb{Z}_{>0}$, only one node is granted access to the channel by the scheduling policy to send its packet. FlexRay uses two different policies that operate in a cyclic fashion to schedule the transmissions, as we explain in Section 3.

Our objective is to provide conditions on the observer and the network, in particular on the scheduling rules and the MATIs associated to each segment, under which the state of observer (2) (approximately) converges to the state of plant (1).

\section{$3 \quad$ FlexRay}

In this section, we briefly present FlexRay and the assumptions we make on the network; for more details, see (Wang et al. 2015). FlexRay works with pre-set communication cycles of length $T>0$. Each cycle contains a static segment of length $T_{1}>0$, a dynamic segment of length $T_{2}>0$ and two protocol segments called symbol window and network idle time, see (Consortium 2005). The lengths of the protocol segments can be considered as negligible compared to $T_{1}$ and $T_{2}$, and hence we ignore them in the network modeling, which allows us to write $T=T_{1}+T_{2}$.

Distinct network access techniques are applied for the static and the dynamic segments. In particular, the time division multiple access (TDMA) approach is employed for the static segment and the nodes get access to the network in a prefixed manner. The static segment consists of time slots of equal length. The chosen node codes its information into packets and then sends them over the communication channel at the beginning of each time slot. The dynamic segment on the other hand uses the flexible time division multiple access (FTDMA) technique (Cena \& Valenzano 2006), and is composed of minislots, which are substantially shorter than static time slots. A dynamic protocol is used to assign priority among the nodes based on the online information. 
The priority can be assigned in a centralized manner, the node with highest priority is assigned with the 'earliest' minislot and the minislots are idle when no nodes compete for access.

We further make the following assumptions on the static and the dynamic segments as in (Wang et al. 2015).

\section{Assumption 1}

(1) Data are transmitted instantaneously and a transmission occurs at the end of the two segments.

(2) The static time slots are of length $\tau_{\text {MATI }}^{s}>0$, where $\tau_{\text {MATI }}^{s}$ is such that $N_{1} \tau_{\text {MATI }}^{s}=T_{1}$ with $N_{1} \in \mathbb{Z}_{>0}$.

(3) The inter-transmission time interval in the dynamic segments, denoted by $\tau_{d y, i}$, satisfies $\tau_{d y, i} \in\left[\tau_{\min }, \tau_{\text {MATI }}^{d}\right]$, where $\tau_{\text {MATI }}^{d}$ is the MATI associated with the dynamic segment and $\tau_{\min } \in$ $\left(0, \min \left\{\tau_{\mathrm{MATI}}^{s}, \tau_{\mathrm{MATI}}^{d}\right\}\right]$ refers to the length of a minislot which satisfies $N_{2} \tau_{\min }=T_{2}$ with $N_{2} \in \mathbb{Z}_{>0}$.

We next introduce two clock variables, which are useful to obtain a hybrid model of NCS with FlexRay in the formalism of (Goebel et al. 2012). We introduce $\tau_{1} \in \mathbb{R}_{>0}$ to model the time elapsed since the last transmission, and $\tau_{2} \in \mathbb{R}_{>0}$ to describe the time elapsed since the last segment switch. We also introduce a state variable $q \in\{1,2\}$ which keeps track of whether we are in the static segment $(q=1)$ or in the dynamic segment $(q=2)$. Then, the transmission and segments switching mechanisms can be modeled as

$$
\left.\begin{array}{r}
\left.\begin{array}{r}
\dot{\tau}_{1}=1 \\
\dot{\tau}_{2}=1 \\
\dot{q}=0
\end{array}\right\} \quad\left(\tau_{1}, \tau_{2}, q\right) \in C_{1} \cup C_{2} \\
\tau_{1}^{+}=0 \\
\tau_{2}^{+}=\tau_{2} \\
q^{+}=q
\end{array}\right\} \quad\left(\tau_{1}, \tau_{2}, q\right) \in D_{1}^{\text {trans }} \cup D_{2}^{\text {trans }}
$$

where

$$
\begin{aligned}
C_{1} & :=\left[0, \tau_{\text {MATI }}^{s}\right] \times\left[0, T_{1}\right] \times\{1\} \\
C_{2} & :=\left[0, \tau_{\text {MATI }}^{d}\right] \times\left[0, T_{2}\right] \times\{2\} \\
D_{1}^{\text {trans }} & :=\left\{\tau_{\text {MATI }}^{s}\right\} \times\left[0, T_{1}\right] \times\{1\} \\
D_{2}^{\text {trans }} & :=\left[\tau_{\text {min }}, \tau_{\text {MATI }}^{d}\right] \times\left[0, T_{2}\right] \times\{2\} \\
D_{1}^{\text {seg }} & :=\{0\} \times\left\{T_{1}\right\} \times\{1\} \\
D_{2}^{\text {seg }} & :=\{0\} \times\left\{T_{2}\right\} \times\{2\} .
\end{aligned}
$$

We can see from (3b) that a transmission jump, corresponding to $\tau_{1}$ being reset to 0 , occurs when $\tau_{1}$ reaches $\tau_{\text {MATI }}^{s}$ during the static segment $(q=1)$, and is allowed to occur whenever $\tau_{1} \in\left[\tau_{\text {min }}, \tau_{\text {MATI }}^{d}\right]$ during the dynamic segment $(q=2)$. On the other hand, a segment jump, corresponding to $\tau_{2}$ being reset to 0 and $q$ being toggled to $3-q$, occurs when $\tau_{2}$ reaches $T_{1}$ when $q=1$ and $T_{2}$ when $q=2$. In this case, $\tau_{1}$ has to be equal to 0 , which corresponds to a transmission at the end of each segment, which is in agreement with item (1) in Assumption 1. Otherwise, the solutions to (3) will stop to exist, which is not a problem in the subsequent analysis. Hence, only the (maximal) solutions, which satisfy the second part of item (1) of Assumption 1, are complete.

\section{Observer Emulation}

We emulate observer (2) as follows

$$
\dot{x}_{o}=\mathbf{f}_{o}\left(x_{o}, \hat{u}, \hat{y}_{p}-\hat{y}_{o}\right) \text {. }
$$

As already mentioned in Section 2, the emulated observer (4) no longer depends on $\left(y_{p}, u\right)$, but on $\left(\hat{y}_{p}, \hat{u}\right)$ because of the network. These variables are generated by the observer based on the received data. Furthermore, observer (4) does not depend on its own output $y_{o}$, as in (2), but on $\hat{y}_{o}$. The variable $\hat{y}_{o}$ is an artificially introduced networked version of $y_{o}$. The idea to use $\hat{y}_{o}$ instead of $y_{o}$ was suggested in (Postoyan et al. 2014) and allows ensuring stronger stability properties for the estimation error system, see Section VIII in (Postoyan et al. 2014). We next present the dynamics of $\hat{y}_{p}$ and $\hat{u}$ before proceeding with the construction of the dynamics of $\hat{y}_{o}$.

Let $\left(y_{p}, u\right)=\left(y_{p_{1}}, \cdots, y_{p_{n y}}, u_{1}, \cdots, u_{n_{u}}\right)$ and $\left(\hat{y}_{p}, \hat{u}\right)=$ $\left(\hat{y}_{p_{1}}, \cdots, \hat{y}_{p_{n_{y}}}, \hat{u}_{1}, \cdots, \hat{u}_{n_{u}}\right)$, where $n_{y}, n_{u} \in \mathbb{Z}_{>0}$. Suppose that node $j \in\{1, \cdots, \ell\}$ is selected by the protocol at time $t_{i}, i \in \mathbb{Z}_{>0}$, and say that the components $y_{p_{j_{y}}}$ and $u_{j_{u}}$ of $y_{p}$ and $u$, respectively, are associated to node $j$, with $j_{y} \in\left\{1, \cdots, n_{y}\right\}$ and $j_{u} \in\left\{1, \cdots, n_{u}\right\}$. Then

$$
\hat{y}_{p_{j y}}\left(t_{i}^{+}\right)=y_{p_{j_{y}}}\left(t_{i}\right) \quad \hat{u}_{j_{u}}\left(t_{i}^{+}\right)=u_{j_{u}}\left(t_{i}\right),
$$

while for all other components of $\hat{y}_{p}$ and $\hat{u}$,

$$
\hat{y}_{p_{k_{y}}}\left(t_{i}^{+}\right)=\hat{y}_{p_{k_{y}}}\left(t_{i}\right) \quad \hat{u}_{k_{u}}\left(t_{i}^{+}\right)=\hat{u}_{k_{u}}\left(t_{i}\right),
$$

with $k_{y} \in\left\{1, \cdots, n_{y}\right\}$ and $k_{u} \in\left\{1, \cdots, n_{u}\right\}$ satisfying $k_{y} \neq j_{y}$ and $k_{u} \neq j_{u}$. We can see from (6) that the components of $\hat{y}$ and $\hat{u}$ corresponding to the $j$-th node are updated and the other components are kept unchanged. For simplicity, we use zero-order-hold devices to implement the observer which gives $\dot{\hat{y}}_{p}=0$ and $\dot{\hat{u}}=0$ for $t \in\left[t_{i}, t_{i+1}\right]$ and $i \in \mathbb{Z}_{>0}$, however other choices are possible.

The variable $\hat{y}_{o}$ is constructed to evolve along the same vector field as $\hat{y}_{p}$ between two successive transmission instants, i.e., $\dot{\hat{y}}_{o}=0$ for $t \in\left[t_{i}, t_{i+1}\right]$. We now explain how $\hat{y}_{o}$ jumps at each transmission instant. Let $y_{o}=\left(y_{o_{1}}, \cdots, y_{o_{n_{y}}}\right)$ and $\hat{y}_{o}=\left(\hat{y}_{o_{1}}, \cdots, \hat{y}_{o_{n_{y}}}\right)$. At each transmission of a component of $\hat{y}_{p}$, say $\hat{y}_{p_{j_{y}}}$ with $j_{y} \in$ 
$\left\{1, \ldots, n_{y}\right\}$, the corresponding component of $\hat{y}_{o}$, that is $\hat{y}_{o_{y}}$, is reset to $y_{o_{j_{y}}}$, which gives

$$
\hat{y}_{o_{j}}\left(t_{i}^{+}\right)=\left\{\begin{array}{l}
y_{o_{y}}\left(t_{i}\right) \text { if } \hat{y}_{p_{j_{y}}}\left(t_{i}^{+}\right)=y_{p_{j y}}\left(t_{i}\right) \\
\hat{y}_{o_{j y}}\left(t_{i}\right) \text { otherwise. }
\end{array}\right.
$$

In view of (7), the dynamics of $\hat{y}_{o}$, loosely speaking, mimics the dynamics of $\hat{y}_{p}$.

For the sake of convenience, we introduce the networkinduced error on the plant output $e_{p}:=\hat{y}_{p}-y_{p} \in \mathbb{R}^{n_{e}}$, $n_{e}:=n_{y}$, and the network-induced error on the input $e_{u}:=\hat{u}-u \in \mathbb{R}^{n_{u}}$. We also define an artificially introduced network-induced error on the observer output $e_{o}:=\hat{y}_{o}-y_{o} \in \mathbb{R}^{n_{e}}$. Using the definitions of $e_{p}, e_{u}$ and $e_{o}$, we can rewrite (4) as

$$
\dot{x}_{o}=\mathbf{f}_{o}\left(x_{o}, u+e_{u}, y_{p}-y_{o}+e\right),
$$

where $e:=e_{p}-e_{o}$ corresponds to the network-induced error on the observation error $y_{p}-y_{o}$.

\section{Switched Protocols}

In this section, we model the transmission mechanisms of FlexRay under the assumptions made in Section 3 and we present the class of scheduling rules we consider.

\subsection{Model}

The network-induced errors introduced in the previous section are useful to model the scheduling mechanism of FlexRay. Let $e_{p}=\left(e_{p_{1}}, \cdots, e_{p_{n_{e}}}\right)$, $e_{o}=\left(e_{o_{1}}, \cdots, e_{o_{n_{e}}}\right)$ and $e_{u}=\left(e_{u_{1}}, \cdots, e_{u_{n_{u}}}\right)$. If the node $j$ associated to $y_{p_{j}}$ and $u_{j_{u}}$ (see Section 4) has access to the network, it follows from (5), (6) and (7) that $e_{p_{j_{y}}}\left(t_{i}^{+}\right)=\hat{y}_{p_{j_{y}}}\left(t_{i}^{+}\right)-y_{p_{j_{y}}}\left(t_{i}\right)=0$ with $j_{y} \in\left\{1, \cdots, n_{e}\right\}$ and $e_{p_{k y}}\left(t_{i}^{+}\right)=\hat{y}_{p_{k_{y}}}\left(t_{i}^{+}\right)-y_{p_{k y}}\left(t_{i}\right)=e_{p_{k y}}\left(t_{i}\right)$ for $k_{y} \in\left\{1, \cdots, n_{e}\right\}$ satisfying $k_{y} \neq j_{y}$. Note that similar dynamics properties also hold for $e_{o}$ and $e_{u}$ by following the same lines. We then model the dynamics of $e_{p}, e_{o}$ and $e_{u}$ at each transmission time using the general form $e_{p}\left(t_{i}^{+}\right)=h_{p}\left(i, e_{p}\left(t_{i}\right), e_{u}\left(t_{i}\right), e_{o}\left(t_{i}\right), q\left(t_{i}\right)\right)$, $e_{o}\left(t_{i}^{+}\right)=h_{o}\left(i, e_{p}\left(t_{i}\right), e_{u}\left(t_{i}\right), e_{o}\left(t_{i}\right), q\left(t_{i}\right)\right)$ and $e_{u}\left(t_{i}^{+}\right)=$ $h_{u}\left(i, e_{p}\left(t_{i}\right), e_{u}\left(t_{i}\right), e_{o}\left(t_{i}\right), q\left(t_{i}\right)\right)$. The functions $h_{p}, h_{o}$ and $h_{u}$ are assumed to be continuous functions; an example is provided in Section 5.2. We then derive that

$$
e\left(t_{i}^{+}\right)=h_{e}\left(i, e_{p}\left(t_{i}\right), e\left(t_{i},\right), e_{u}\left(t_{i}\right), q\left(t_{i}\right)\right),
$$

where $h_{e}$ is obtained from $h_{p}$ and $h_{o}$, noting that we make it depend on $\left(i, e_{p}, e, e_{u}, q\right)$ and not $\left(i, e_{p}, e_{u}, e_{o}, q\right)$, which is equivalent in view of the definition of $e$. In view of (8), the dynamics of the observer is only affected by $e$ and $e_{u}$. We therefore introduce the variable $\mathbf{e}:=\left(e, e_{u}\right)$. In that way, we term the equation below,

$$
\mathbf{e}\left(t_{i}^{+}\right)=h_{\mathbf{e}}\left(i, e_{p}\left(t_{i}\right), \mathbf{e}\left(t_{i}\right), q\left(t_{i}\right)\right),
$$

which specifies how e at transmission time $t_{i}$ is mapped at $t_{i}^{+}$as the protocol equation, where $h_{\mathbf{e}}$ is continuous since so are $h_{p}, h_{u}$ and $h_{o}$. Recall that $q: \mathbb{R}_{\geq 0} \rightarrow\{1,2\}$ is a switching signal indicating which segment is active. The map $h_{\mathbf{e}}$ has the form of

$$
\begin{gathered}
h_{\mathbf{e}}\left(i, e_{p}\left(t_{i}\right), \mathbf{e}\left(t_{i}\right), q\left(t_{i}\right)\right):=\left(2-q\left(t_{i}\right)\right) h_{\mathbf{e}, 1}\left(i, e_{p}\left(t_{i}\right), \mathbf{e}\left(t_{i}\right)\right) \\
+\left(q\left(t_{i}\right)-1\right) h_{\mathbf{e}, 2}\left(i, e_{p}\left(t_{i}\right), \mathbf{e}\left(t_{i}\right)\right),
\end{gathered}
$$

where $h_{\mathbf{e}, 1}$ is the scheduling rule corresponding to the static segment and $h_{\mathbf{e}, 2}$ corresponds to the dynamic segment.

\subsection{Assumption}

We assume that the scheduling rule (10), which governs the transmissions of the static and the dynamic segments, satisfies the properties listed below.

Assumption 2 For each $m \in\{1,2\}$, there exist $W_{m}$ : $\mathbb{R}^{n_{e}+n_{u}} \times \mathbb{Z}_{\geq 0} \rightarrow \mathbb{R}_{\geq 0}$, which is locally Lipschitz in its first argument, $\sigma_{m}^{v} \in \mathcal{K}_{\infty}$, constants $\underline{\alpha}_{W_{m}}, \bar{\alpha}_{W_{m}}>0$ and $\rho_{m} \in(0,1)$ such that for any $\kappa \in \mathbb{Z}_{\geq 0}, e_{p} \in \mathbb{R}^{n_{e}}$, $\mathbf{e} \in \mathbb{R}^{n_{e}+n_{u}}$ and $v \in \mathbb{R}^{n_{e}}$ :

(1) $\underline{\alpha}_{W_{m}}|\mathbf{e}| \leq W_{m}(\mathbf{e}, \kappa) \leq \bar{\alpha}_{W_{m}}|\mathbf{e}|$,

(2) $W_{m}\left(h_{\mathbf{e}, m}\left(\kappa, e_{p}, \mathbf{e}\right), \kappa+1\right) \leq \rho_{m} W_{m}(\mathbf{e}, \kappa)+\sigma_{m}^{v}(|v|)$.

Assumption 2 is a stability condition on the protocol equation (10). The underlying idea is to treat protocols as discrete-time dynamical systems as suggested in (Nešić \& Teel 2004), which appears to be extremely useful to study NCS stability. More precisely, Assumption 2 states that (10) is an (exponentially) input-to-state stable (ISS) protocol, as termed in (Tabbara \& Nešić 2008). We next provide an example of a switched protocol that satisfies these conditions.

Example 1 We consider a switched protocol which employs the $R R$ scheduling rule during the static segment and a TOD-like protocol during the dynamic segment. $R R$ grants access to the nodes in a periodic fashion and it follows from (Nešić \& Teel 2004) that $h_{\mathbf{e}, 1}\left(i, e_{p}, \mathbf{e}\right):=(I-\Delta(i)) \mathbf{e}$, where $i \in \mathbb{Z}_{>0}$, $\Delta(i)=\operatorname{diag}\left\{\tilde{\delta}_{1}(i) I_{n_{1}}, \cdots, \tilde{\delta}_{\ell}(i) I_{n_{\ell}}\right\}, I_{n_{s}}$ is the identity matrix of dimension $n_{s}$ with $\sum_{s=1}^{\ell} n_{s}=n_{e}, \tilde{\delta}_{s}$ satisfies $\tilde{\delta}_{s}(i)=1$ when $i=s+k \ell$ for $k \in \mathbb{Z}_{\geq 0}$ and $\tilde{\delta}_{s}(i)=0$ otherwise, for $s \in\{1, \ldots, \ell\}$ and $i \in \mathbb{Z}_{\geq 0}^{-}$.

On the other hand, TOD gives access to the node with the largest mismatch between the current signal value and the last transmitted one (Walsh $\&$ Ye 2001). We partition $\mathbf{e}$ as $\mathbf{e}=\left(\mathbf{e}_{1}, \cdots, \mathbf{e}_{\ell}\right)$ and then a TOD-like protocol from (Postoyan et al. 2014) grants access to the node where $\left|\mathbf{e}_{j}\right|$ is the biggest for $j \in\{1, \cdots, \ell\}$. Then, $h_{\mathbf{e}, 2}\left(i, e_{p}, \mathbf{e}\right):=$ $(I-\Psi(\mathbf{e})) \mathbf{e}$, where $\Psi(\mathbf{e}):=\operatorname{diag}\left\{\psi_{1}(\mathbf{e}) I_{n_{1}}, \psi_{2}(\mathbf{e}) I_{n_{2}}\right.$, $\left.\cdots, \psi_{\ell}(\mathbf{e}) I_{n_{\ell}}\right\}$. The function $\psi_{s}$ satisfies $\psi_{s}(\mathbf{e})=1$ when 
$s=\min \left(\arg \max _{j \in\{1, \cdots, \ell\}}\left|\mathbf{e}_{j}\right|\right)$ and $\psi_{s}(\mathbf{e})=0$ otherwise, for $s \in\{1, \cdots, \ell\}$ and $i \in \mathbb{Z}_{\geq 0}$.

By following similar lines as in Propositions 3-4 in (Postoyan et al. 2014), we have that the switched RRTOD-like protocol satisfies Assumption 2 with $\rho_{1}=\rho_{2}=$ $\sqrt{\frac{\ell-1}{\ell}}, \sigma_{1}^{v}(s)=\sqrt{\ell}\left(1+\rho_{1}\right) s$ and $\sigma_{2}^{v}(s)=\left(1+\rho_{2}\right) s$ for $s \geq 0, \underline{\alpha}_{W_{1}}=1, \bar{\alpha}_{W_{1}}=\sqrt{\ell}, \underline{\alpha}_{W_{2}}=\bar{\alpha}_{W_{2}}=1$, where $W_{1}(\mathbf{e}, \kappa):=\sqrt{\sum_{i=\kappa}^{\kappa+l}|\phi(i, \kappa, \mathbf{e})|^{2}}, W_{2}(\mathbf{e}, \kappa):=|\mathbf{e}|$, $\phi(i, \kappa, \mathbf{e})$ is the solution to $\mathbf{e}(\kappa+1)=h_{\mathbf{e}, 1}\left(\kappa, e_{p}, \mathbf{e}(\kappa)\right)$ at time $i$ with initial condition e and $\kappa$.

\section{$6 \quad$ NCS Model}

We now write the overall system. We introduce for this purpose the variable $\kappa \in \mathbb{Z}_{\geq 0}$ to count the number of transmissions, which is useful to model scheduling policies such as RR. We also introduce the estimation error $\xi:=x_{o}-x_{p} \in \mathbb{R}^{n_{\xi}}$ with $n_{\xi}=n_{p}$, and $d:=$ $\left(d_{u}, d_{v}\right)$, where $d_{u}$ and $d_{v}$ respectively denote the timederivative of the input signal $u$ and of the noise $v$. Let $\psi:=\left(\xi, x_{p}, \mathbf{e}, e_{p}, \kappa, \tau_{1}, \tau_{2}, q\right)$ be the full state vector. In view of Sections 2 to 5 , we model the overall system using the hybrid formalism of (Cai \& Teel 2009, Goebel et al. 2012), which gives

$$
\begin{aligned}
\dot{\psi} & =\mathcal{F}(\psi, u, v, w, d) & & \psi \in C \\
\psi^{+} & =\mathcal{G}(\psi) & & \psi \in D,
\end{aligned}
$$

where $(u, v, w, d) \in \mathbb{R}^{2 n_{u}+n_{w}+2 n_{e}}, C:=\mathbb{R}^{n_{\xi}+n_{p}+2 n_{e}+n_{u}} \times$ $\mathbb{Z}_{\geq 0} \times\left(C_{1} \cup C_{2}\right), D:=\mathbb{R}^{n_{\xi}+n_{p}+2 n_{e}+n_{u}} \times \mathbb{Z}_{>0} \times\left(D_{1} \cup D_{2}\right)$, $D_{1}:=D_{1}^{\text {trans }} \cup D_{1}^{\text {seg }}, D_{2}:=D_{2}^{\text {trans }} \cup D_{2}^{\text {seg }}$, and $D_{1}^{\text {seg }}$, $D_{2}^{\text {seg }}, D_{1}^{\text {trans }}$ and $D_{2}^{\text {trans }}$ come from $(3)$.

The mapping $\mathcal{F}$ in (12) is defined as

$$
\begin{array}{r}
\mathcal{F}(\psi, u, v, w, d):=\left(f_{\xi}(\psi, u, v, w), f_{p}(\psi, u, v, w),\right. \\
\left.g_{\mathbf{e}}(\psi, u, v, w, d), g_{p}(\psi, u, v, w, d), 0,1,1,0\right),
\end{array}
$$

where $f_{\xi}, f_{p}$ are obtained by direct calculations from (1) and $(2), g_{\mathbf{e}}(\psi, u, v, w, d):=\left(g_{e}\left(\psi, u, v, w, d_{v}\right), g_{u}\left(d_{u}\right)\right)$, $g_{e}\left(\psi, u, v, w, d_{v}\right):=\frac{\partial \mathbf{g}}{\partial x_{o}}\left(x_{p}+\xi\right) \mathbf{f}_{o}\left(x_{p}+\xi, u+e_{u}, \mathbf{g}\left(x_{p}\right)+\right.$ $\left.v-\mathbf{g}\left(x_{p}+\xi\right)+e\right)-\frac{\partial \mathbf{g}}{\partial x_{p}}\left(x_{p}\right) \mathbf{f}_{p}\left(x_{p}, u+e_{u}, w\right)-d_{v}, g_{u}\left(d_{u}\right):=$ $-d_{u}, g_{p}(\psi, u, v, w, d):=-\frac{\partial \mathbf{g}}{\partial x_{p}}\left(x_{p}\right) \mathbf{f}_{p}\left(x_{p}, u+e_{u}, w\right)-d_{v}$. We note that the derivatives of the input signal and of the measurement noise do appear in the dynamics of the system.

The mapping $\mathcal{G}(\psi):=\mathcal{G}^{\text {trans }}(\psi)$ when $\left(\tau_{1}, \tau_{2}, q\right) \in$ $D_{1}^{\text {trans }} \cup D_{2}^{\text {trans }}$ and $\mathcal{G}(\psi):=\mathcal{G}^{\operatorname{seg}}(\psi)$ when $\left(\tau_{1}, \tau_{2}, q\right) \in$ $D_{1}^{\text {seg }} \cup D_{2}^{\text {seg }}$, where $\mathcal{G}^{\text {trans }}(\psi):=\left(\xi, x_{p}, h_{\mathbf{e}}\left(\kappa, e_{p}, \mathbf{e}, q\right)\right.$, $\left.h_{p}\left(\kappa, e_{p}, \mathbf{e}, q\right), \kappa+1,0, \tau_{2}, q\right)$ corresponds to a transmission jump, $\mathcal{G}^{\operatorname{seg}}(\psi):=\left(\xi, x_{p}, \mathbf{e}, e_{p}, \kappa, \tau_{1}, 0,3-q\right)$ corresponds to a segment switching jump, $h_{p}$ and $h_{\mathbf{e}}$ come from Section 5.1. We can see that the map $\mathcal{G}$ gen- erates two kinds of jumps whether it corresponds to a transmission or a segment switch.

\section{Stability Analysis}

We first assume an exponential growth condition on the e-subsystem during two consecutive transmissions, like in (Postoyan \& Nešić 2012, Postoyan et al. 2014).

Assumption 3 For each $m \in\{1,2\}$, there exist a continuous function $H_{m}: \mathbb{R}^{n_{\xi}} \rightarrow \mathbb{R}_{\geq 0}, \sigma_{m} \in \mathcal{K}_{\infty}$ and $L_{m} \geq 0$ such that for all $\xi \in \mathbb{R}^{n_{\xi}}, v \in \mathbb{R}^{n_{e}}, w \in \mathbb{R}^{n_{w}}$, $d \in \mathbb{R}^{n_{u}+n_{e}}, \kappa \in \mathbb{Z}_{\geq 0}$ and almost all $\mathbf{e} \in \mathbb{R}^{n_{e}+n_{u}}$ : $\left\langle\frac{\partial W_{m}(\mathbf{e}, \kappa)}{\partial \mathbf{e}}, g_{\mathbf{e}}(\psi, u, v, w, d)\right\rangle \leq L_{m} W_{m}(\mathbf{e}, \kappa)+H_{m}(\xi)+$ $\sigma_{m}(|(u, v, w, d)|)$, where the function $W_{m}$ comes from $A s$ sumption 2.

The above condition is satisfied when $W_{m}$ is globally Lipschitz in e with constants $S_{m} \geq 0$, which is the case for the protocol in Example 1, and when $\left|g_{\mathbf{e}}(\psi, u, v, w, d)\right| \leq$ $S_{3}|\mathbf{e}|+S_{4}|\xi|+S_{5}|(u, v, w, d)|$ for all $(\psi, u, v, w, d) \in$ $\mathbb{R}^{n_{\xi}+n_{p}+2 n_{e}+n_{u}} \times \mathbb{Z}_{\geq 0} \times \mathbb{R}_{\geq 0}^{2} \times\{1,2\} \times \mathbb{R}^{2 n_{u}+2 n_{v}+n_{w}}$, with $S_{3}, S_{4}, S_{5}>0$. In this case, Assumption 3 holds with $L_{m}:=\frac{S_{m} S_{3}}{\underline{\alpha}_{W_{m}}}, H_{m}(\xi):=S_{m} S_{4}|\xi|$ and $\sigma_{m}(s):=S_{m} S_{5} s$ for all $\xi \in \mathbb{R}^{n_{\xi}}$ and $s \geq 0$, where $\underline{\alpha}_{W_{m}}>0$ comes from Example 1.

We next assume that the observer (2) has been designed such that the following conditions hold.

Assumption 4 There exist a continuously differentiable function $V: \mathbb{R}^{n_{\xi}} \rightarrow \mathbb{R}_{\geq 0}$ and $\underline{\alpha}_{V}, \bar{\alpha}_{V} \in \mathcal{K}_{\infty}$ such that for each $m \in\{1,2\}$ there exist $\mu_{m} \in \mathcal{K}_{\infty}$ and $\varepsilon_{m}, \gamma_{m}>0$ such that for all $\xi \in \mathbb{R}^{n_{\xi}}, \mathbf{e} \in \mathbb{R}^{n_{e}+n_{u}}$, $v \in \mathbb{R}^{n_{e}}, w \in \mathbb{R}^{n_{w}}$ and $\kappa \in \mathbb{Z}_{\geq 0}$,

(1) $\underline{\alpha}_{V}(|\xi|) \leq V(\xi) \leq \bar{\alpha}_{V}(|\xi|)$,

(2) $\left\langle\nabla V(\xi), f_{\xi}(\psi, v, w)\right\rangle \leq-\varepsilon_{m} V(\xi)-\varepsilon_{m} W_{m}^{2}(\mathbf{e}, \kappa)-$ $H_{m}^{2}(\xi)+\gamma_{m}^{2} W_{m}^{2}(\mathbf{e}, \kappa)+\mu_{m}(|(u, v, w)|)$, where $W_{m}$ and $H_{m}$ come respectively from Assumptions 2-3. $\square$

Assumption 4 ensures that observer (2) is robust for plant (1). In particular, the $\xi$-system in (12) is $\mathcal{L}_{2}$ stable from $\left(W_{m}, \sqrt{\mu_{m}}\right)$ to $H_{m}$, and ISS with respect to $(u, v, w)$.

We next present the bounds we impose on the MATI of each segment, that is $\tau_{\text {MATI }}^{s}$ and $\tau_{\text {MATI }}^{d}$.

Assumption 5 Given $T_{1}, T_{2}>0, m \in\{1,2\}$, let

$\mathcal{T}_{m}$

$=\left\{\begin{array}{lc}\frac{1}{\tilde{L}_{m}\left(T_{m}\right) r_{m}} \arctan \left(\theta\left(T_{m}\right)\right) & \gamma_{m}>\tilde{L}_{m}\left(T_{m}\right) \\ \frac{1}{\tilde{L}_{m}\left(T_{m}\right)+\rho_{m} \gamma_{m} \lambda_{m}}-\frac{\rho_{m}}{\rho_{m} \tilde{L}_{m}\left(T_{m}\right)+\gamma_{m} \lambda_{m}} & \gamma_{m}=\tilde{L}_{m}\left(T_{m}\right) \\ \frac{1}{\tilde{L}_{m}\left(T_{m}\right) r_{m}} \operatorname{arctanh}\left(\theta\left(T_{m}\right)\right) & \gamma_{m}<\tilde{L}_{m}\left(T_{m}\right)\end{array}\right.$ 
where $\tilde{L}_{m}\left(T_{m}\right):=\frac{1}{2}\left(2 L_{m}+\frac{1}{T_{m}} \ln \left(\lambda_{m}\right)\right)$ and $\lambda_{m}:=$ $\max \left\{1, \frac{\gamma_{3-m} \bar{\alpha}_{W_{3-m}}^{2}}{\gamma_{m} \rho_{1} \rho_{2} \underline{\alpha}_{W_{m}}^{2}}\right\}$ with $L_{m}, \gamma_{m}, \underline{\alpha}_{W_{m}}, \bar{\alpha}_{W_{m}}>0$ and $\rho_{m} \in(0,1)$ coming from Assumptions 2-4, $\theta\left(T_{m}\right):=$ $\frac{r_{m}\left(1-\rho_{m}\right)}{2 \frac{\rho_{m}}{1+\rho_{m}}\left(\frac{\gamma_{m}}{L_{m}\left(T_{m}\right)}-1\right)+1+\rho_{m}}, r_{m}:=\sqrt{\left|\frac{\gamma_{m}^{2}}{L_{m}^{2}}-1\right|}$. The constants $\tau_{\text {MATI }}^{s}$ and $\tau_{\text {MATI }}^{d}$ are such that $\tau_{\text {MATI }}^{s}<\frac{T_{1}}{\left\lceil T_{1} / \mathcal{T}_{1}\right\rceil}$ and $\tau_{\text {MATI }}^{d}<\mathcal{T}_{2}$.

The bounds in (14) depend on constants introduced in Assumptions 2-4 and on the arbitrarily selected segment lengths $T_{1}$ and $T_{2}$. Hence, once Assumptions 2-4 have been verified, we derive the MATI bounds to be implemented using Assumption 5 for any given value of $T_{1}$ and $T_{2}$. We note that the smaller $T_{m}$, the smaller $\mathcal{T}_{m}$. On the other hand, when $T_{1}$ goes to infinity and only one segment is activated (noting that $\tilde{L}_{1}\left(T_{1}\right) \rightarrow L_{1}$ here), $\tau_{\text {MATI } 1}$ corresponds to the MATI in (Postoyan et al. 2014) where observers are designed for NCS with nonswitched protocols.

Remark 1 Contrary to the MATI bounds in (Wang et al. 2015) where the corresponding stabilization problem was studied, the expression in Assumption 5 exclusively depends on parameters coming from Assumptions 2-4, which make the designs of $\tau_{\mathrm{MATI}}^{s}$ and $\tau_{\mathrm{MATI}}^{d}$ easier.

All the required assumptions have been stated, we can now present the stability property of the observer. The proof of the next theorem is provided in the appendix.

Theorem 1 Suppose Assumptions 2-5 hold for system (12). Then, there exist $\beta \in \mathcal{K} \mathcal{L}$ and $\gamma \in \mathcal{K}_{\infty}$ such that for each solution pair ${ }^{1}(\psi, u, v, w, d)$ to system (12) and for all $(t, j) \in \operatorname{dom}(\psi, u, v, w, d),|\psi(t, j)|_{\mathcal{A}} \leq$ $\beta\left(|\psi(0,0)|_{\mathcal{A}}, t+j\right)+\gamma\left(\|(u, v, w, d)\|_{(t, j)}\right)$, where $\mathcal{A}:=\{\psi: \xi=0, \mathbf{e}=0\}, \gamma(s):=\frac{1}{1-\exp \left(-\varrho \tau_{\min }\right)} \underline{\alpha}_{U} \circ$ $\left(\Delta_{\mathcal{F}}(s)+\widetilde{\varrho} \Delta_{\mathcal{G}}(s)\right)$ for some $\underline{\alpha}_{U}, \Delta_{\mathcal{F}}, \Delta_{\mathcal{G}} \in \mathcal{K}_{\infty}$ and $\widetilde{\varrho}>0$.

We can see from Theorem 1 that the estimation error $\xi$ and the network-induced error e converge to a ball centered at the origin and whose radius depends on the $\mathcal{L}_{\infty}$ norm of the input $(u, v, w, d)$. We can also see from Theorem 1 that $\xi$ and e do not a priori converge to the origin even when $w=0$ and $v=0$ since, in this case, $|(\xi(t, j), \mathbf{e}(t, j))| \leq \beta(|(\xi(0,0), \mathbf{e}(0,0))|, t+j)+$ $\gamma\left(\left\|d_{u}\right\|_{(t, j)}\right)$. The nonlinear ISS gain $\gamma$ in Theorem 1 depends on $\tau_{\text {min }}$, which is the minimum time between two tranmission jumps during the dynamic segment. We can also see that $\gamma$ goes to infinity when $\tau_{\text {min }}$ approaches zero. This is due to our stability analysis which requires

\footnotetext{
1 See (Cai \& Teel 2009) for a definition of the notion of solution pair to system (12) as well as for a definition of the $\mathcal{L}_{\infty}$ norm used in Theorem 1.
}

the Lyapunov function to decrease for some time, at least $\tau_{\text {min }}$, during flows in order to compensate a possible increase at jump, see details in the proof of Theorem 1 given in the appendix.

\section{Conclusions}

We proposed an observer design approach for nonlinear NCS with FlexRay. FlexRay is composed of communications cycles and each cycle consists of a static and a dynamic segment for which different scheduling policies apply. For an observer designed in the absence of communication constraints, we investigated the conditions on the network to preserve its convergence property. In particular, we provided explicit segment-dependent maximal allowable transmission interval (MATI) bounds and showed that the estimation error verifies an input-tostate stable (ISS) property, provided that MATI bounds are satisfied and scheduling rules are ISS.

\section{References}

Cai, C. \& Teel, A. R. (2009), 'Characterizations of inputto-state stability for hybrid systems', Systems $\&$ Control Letters 58, 47-53.

Cena, G. \& Valenzano, A. (2006), 'On the properties of the flexible time division multiple access technique', IEEE Trans. on Industrial Informatics 2, 86-94.

Consortium, F. (2005), FlexRay Communications System - Protocol Specification V2.1 Rev.A.

Goebel, R., Sanfelice, R. G. \& Teel, A. R. (2012), Hybrid dynamical systems: Modeling, Stability, and Robustness, Princeton University Press.

Naghshtabrizi, P. \& Hespanha, J. P. (2009), Analysis of distributed control systems with shared communication and computation resources, in 'Proceedings of the American control conference', Saint Louis, USA, pp. 3384-3389.

Nešić, D. \& Teel, A. R. (2004), 'Input-output stability properties of networked control systems', IEEE Transactions on Automatic Control 49, 1650-1667.

Postoyan, R. \& Nešić, D. (2012), 'A framework for the observer design for networked control systems', IEEE Trans. on Aut. Contr. 57, 1309-1314.

Postoyan, R., van de Wouw, N., Nešić, D. \& Heemels, W. (2014), 'Tracking control for nonlinear networked control systems', IEEE Trans. on Aut. Contr. 59, 15391554 .

Schmidt, E. G. \& Schmidt, K. (2009), 'Message scheduling for the Flexray protocol: the dynamic segment', IEEE Trans. on Vehicular Tech. 58, 2160-2169.

Tabbara, M. \& Nešić, D. (2008), Input-output stability with input-to-state stable protocols for quantized and networked control systems, in 'Proceedings of the 47th IEEE CDC', Cancun, Maxico, pp. 2680-2685.

Teel, A. R. \& Praly, L. (2000), 'On assigning the derivative of a disturbance attenuation control lyapunov function', Math. Control Signals System 13, 95-124. 
Walsh, G. C. \& Ye, H. (2001), 'Scheduling of networked control systems', IEEE Control Systems Magzine 21, 57-65.

Wang, W., Nešić, D. \& Postoyan, R. (2015), 'Emulationbased stabilization of networked control systems implemented on Flexray', Automatica 59, 73-83.

\section{A Proof of Theorem 1}

We need the following two technical lemmas to show the main results. Lemma 1 follows from the same lines as the proof of Lemma 3 in (Wang et al. 2015) and Lemma 2 can be derived according to the second paragraph of the proof of Theorem 1 in (Postoyan et al. 2014), using Lemma 1. Hence, their proofs are omitted.

Lemma 1 The solution $\bar{\phi}_{m}, m \in\{1,2\}$, of $\frac{d \bar{\phi}_{m}}{d s}=$ $-2 \tilde{L}_{m}\left(T_{m}\right) \bar{\phi}_{m}-\gamma_{m} \lambda_{m} \bar{\phi}_{m}^{2}-\frac{\gamma_{m}}{\lambda_{m}}$ with $\bar{\phi}_{m}(0)=\frac{1}{\rho_{m}}$ satisfies $\bar{\phi}_{m}(s) \in\left[\rho_{m}, 1 / \rho_{m}\right]$ for all $s \in\left[0, \mathcal{T}_{m}\right]$, where $\tilde{L}_{m}\left(T_{m}\right), \lambda_{m}$ and $\mathcal{T}_{m}$ come from (14), $\gamma_{m}>0$ from Assumption 4 and $T_{m}>0$.

The bounds $\mathcal{T}_{m}$ on the MATIs in Assumption 5 actually correspond to the time it takes for $\bar{\phi}_{m}$ to decrease from $1 / \rho_{m}$ to $\rho_{m}$ in Lemma 1 .

Lemma 2 Suppose Assumption 5 holds and let $\tau_{\text {MATI }}^{s}<$ $\frac{T_{1}}{\left\lceil T_{1} / \mathcal{T}_{1}\right\rceil}$ and $\tau_{\text {MATI }}^{d}<\mathcal{T}_{2}$ be given. Then, there exist $\rho_{m}^{*} \in\left(\rho_{m}, 1\right)$ and $\eta_{m} \in\left(0, \eta_{m}^{*}\right)$, where $\eta_{m}^{*}:=\left(\frac{\rho_{m}^{*}}{\rho_{m}}\right)^{2}-1$, such that the solution $\phi_{m}$ to $\frac{d \phi_{m}}{d s}=-2 \tilde{L}_{m}\left(T_{m}\right) \phi_{m}-$ $\gamma_{m} \lambda_{m}\left(1+\eta_{m}\right) \phi_{m}^{2}-\frac{\gamma_{m}}{\lambda_{m}}$ with $\phi_{m}(0)=\frac{1}{\rho_{m}^{*}}$ satisfies $\phi_{m}(s) \in\left[\rho_{m}^{*}, 1 / \rho_{m}^{*}\right]$ for all $s \in\left[0, \tau_{\text {MATI }}^{s}\right]$ when $m=1$ and all $s \in\left[0, \tau_{\text {MATI }}^{d}\right]$ when $m=2$.

Let $T_{1}, T_{2}>0$ be given. Let $\rho_{1}^{*}, \rho_{2}^{*}, \eta_{1}^{*}, \eta_{2}^{*}>0$, functions $\phi_{1}$ and $\phi_{2}$ come from Lemma 2 . We consider the Lyapunov function, for $\psi \in C \cup D$

$$
\begin{aligned}
U(\psi):= & V(\xi)+\gamma_{1} \phi_{1}\left(\tau_{1}\right) \vartheta_{1}\left(\tau_{2}\right) W_{1}^{2}(\mathbf{e}, \kappa)(2-q) \\
& +\gamma_{2} \phi_{2}\left(\tau_{1}\right) \vartheta_{2}\left(\tau_{2}\right) W_{2}^{2}(\mathbf{e}, \kappa)(q-1),
\end{aligned}
$$

where $W_{1}$ and $W_{2}$ come from Assumption 2, $V$ from Assumption $4, \vartheta_{m}(s):=\exp \left(\bar{\lambda}_{m} s\right)$ for $s \geq 0, m \in\{1,2\}$, and $\bar{\lambda}_{m}:=1 / T_{m} \cdot \ln \left(\lambda_{m}\right)>0$. The functions $\phi_{m}$ and $\vartheta_{m}$ in (A.1) are constructed differently compared to (Wang et al. 2015), which allows us to derive simpler expressions of the MATI in Assumption 5, as mentioned in Remark 1. We note that $U$ is locally Lipschitz in $\xi, x_{p}, \mathbf{e}, \tau_{1}$ and $\tau_{2}$ in view of Assumptions 2-4 and the definitions of $\phi_{m}$ in Lemma 2.

We next show that there exist $\underline{\alpha}_{U}, \bar{\alpha}_{U} \in \mathcal{K}_{\infty}, \Delta_{\mathcal{F}}, \Delta_{\mathcal{G}} \in$ $\mathcal{K}_{\infty}$ and $\widetilde{\varrho}>0$ such that the following properties hold.

(P1) For all $\psi \in C \cup D, \underline{\alpha}_{U}\left(|\psi|_{\mathcal{A}}\right) \leq U(\psi) \leq \bar{\alpha}_{U}\left(|\psi|_{\mathcal{A}}\right)$.
(P2) For all ${ }^{2} \psi \in C, v \in \mathbb{R}^{n_{e}}, w \in \mathbb{R}^{n_{w}}$ and $d \in \mathbb{R}^{n_{e}+n_{u}}, U^{\circ}(\psi ; \mathcal{F}(\psi, u, v, w, d)) \leq-\widetilde{\varrho} U(\psi)+$ $\Delta_{\mathcal{F}}(|(u, v, w, d)|)$.

(P3) For all $\psi \in D$ and $v \in \mathbb{R}^{n_{e}}, U\left(\mathcal{G}^{\text {trans }}(\psi)\right) \leq U(\psi)+$ $\Delta_{\mathcal{G}}(|v|)$ and $U\left(\mathcal{G}^{\operatorname{seg}}(\psi)\right) \leq U(\psi)$.

From the definition of $\vartheta_{m}, m \in\{1,2\}$, Lemma 2 and Assumptions 2-4, we have that $\underline{\alpha}_{U}\left(|\psi|_{\mathcal{A}}\right) \leq U(\psi) \leq$ $\bar{\alpha}_{U}\left(|\psi|_{\mathcal{A}}\right)$, where $\underline{\alpha}_{U}: s \mapsto \min \left\{\underline{\alpha}_{V}\left(\frac{s}{2}\right), \gamma_{1} \rho_{1}^{*} \underline{\alpha}_{W_{1}}^{2} s^{2}\right.$, $\left.\gamma_{2} \rho_{2}^{*} \underline{\alpha}_{W_{2}}^{2} s^{2}\right\}$ and $\bar{\alpha}_{U}(s): s \mapsto \max \left\{2 \bar{\alpha}_{V}(s), \frac{2 \gamma_{1} \lambda_{1} \bar{\alpha}_{W_{1}}^{2}}{\rho_{1}^{*}} s^{2}\right.$, $\left.\frac{2 \gamma_{2} \lambda_{2} \bar{\alpha}_{W_{2}}^{2}}{\rho_{2}^{*}} s^{2}\right\} \in \mathcal{K}_{\infty}$. The constructions of $\bar{\alpha}_{U}$ and $\underline{\alpha}_{U}$ use the same technique as the proof of Proposition 2 in (Wang et al. 2015).

We next consider (P2). We omit below the dependence of $\phi_{1}$ on $\tau_{1}$ and $\vartheta_{1}$ on $\tau_{2}$ for the sake of convenience. In view of page 99 in (Teel \& Praly 2000) and the definition of $\vartheta_{1}$, for all $\psi \in C$ with $q=1$ and any $(u, v, w, d) \in$ $\mathbb{R}^{2 n_{u}+n_{w}+2 n_{e}}$,

$$
\begin{aligned}
& U^{\circ}(\psi ; \mathcal{F}(\psi, u, v, w, d))=\left\langle\nabla V(\xi), f_{\xi}(\psi, v, w)\right\rangle \\
& +\gamma_{1}\left(\frac{d \phi_{1}}{d \tau_{1}} \vartheta_{1}+\phi_{1} \frac{d \vartheta_{1}}{d \tau_{2}}\right) W_{1}^{2}(\mathbf{e}, \kappa) \\
& +2 \gamma_{1} \phi_{1} \vartheta_{1} W_{1}(\mathbf{e}, \kappa) W_{1}^{\circ}\left(\mathbf{e} ; g_{\mathbf{e}}(\psi, u, v, w, d)\right) \\
& \leq-\varepsilon_{1} V(\xi)-\varepsilon_{1} W_{1}^{2}(\mathbf{e}, \kappa)-H_{1}^{2}(\xi)+\mu_{1}(|(u, v, w)|) \\
& +\gamma_{1}^{2} W_{1}^{2}(\mathbf{e}, \kappa)+\gamma_{1}\left(\frac{d \phi_{1}}{d \tau_{1}}+\phi_{1} \bar{\lambda}_{1}\right) \vartheta_{1} W_{1}^{2}(\mathbf{e}, \kappa)+2 \gamma_{1} \\
& \cdot \phi_{1} \vartheta_{1} W_{1}(\mathbf{e}, \kappa)\left(L_{1} W_{1}(\mathbf{e}, \kappa)+H_{1}(\xi)+\sigma_{1}(|(u, v, w, d)|)\right) .
\end{aligned}
$$

We have $2 \gamma_{1} \phi_{1} \vartheta_{1} W_{1}(\mathbf{e}, \kappa) H_{1}(\xi) \leq \gamma_{1}^{2} \phi_{1}^{2} \vartheta_{1}^{2} W_{1}^{2}(\mathbf{e}, \kappa)$ $+H_{1}^{2}(\xi)$. Hence, in view of $(\mathrm{A} . \overline{2})$, the facts that $\vartheta_{1}\left(\tau_{2}\right) \leq \lambda_{1}$ for all $\tau_{2} \in\left[0, T_{1}\right]$, and $2 \gamma_{1} \phi_{1} \vartheta_{1} W_{1}(\mathbf{e}, \kappa)$ $\sigma_{1}(|(u, v, w, d)|) \leq \eta_{1} \gamma_{1}^{2} \phi_{1}^{2} \vartheta_{1}^{2} W_{1}^{2}(\mathbf{e}, \kappa)+\frac{1}{\eta_{1}} \sigma_{1}^{2}(|(u, v, w, d)|)$ for $\eta_{1}>0$ from Lemma 2 ,

$$
\begin{aligned}
& U^{\circ}(\psi ; \mathcal{F}(\psi, u, v, w, d)) \leq-\varepsilon_{1} V(\xi)-\varepsilon_{1} W_{1}^{2}(\mathbf{e}, \kappa) \\
& +\underbrace{\gamma_{1} \lambda_{1}\left(\frac{d \phi_{1}}{d \tau_{1}}+\gamma_{1} \lambda_{1}\left(1+\eta_{1}\right) \phi_{1}^{2}+\left(2 L_{1}+\bar{\lambda}_{1}\right) \phi_{1}+\frac{\gamma_{1}}{\lambda_{1}}\right)}_{(a)} \\
& \cdot W_{1}^{2}(\mathbf{e}, \kappa)+\Delta_{\mathcal{F}}(|(u, v, w, d)|)
\end{aligned}
$$

where $\Delta_{\mathcal{F}}(s):=\mu_{1}(s)+\frac{1}{\eta_{1}} \sigma_{1}^{2}(s)$ for each $s \geq 0$. Note that $\tilde{L}_{1}\left(T_{1}\right)=\frac{1}{2}\left(2 L_{1}+\bar{\lambda}_{1}\right)$ since $\bar{\lambda}_{1}=\frac{\ln \lambda_{1}}{T_{1}}$. Then, in view of Lemma 2 , the items subscripted by (a) in (A.3) equal to zero when $\psi \in C$. Then, from (A.1), $U^{\circ}(\psi ; \mathcal{F}(\psi, u, v, w, d)) \leq-\varepsilon_{1} V(\xi)-\varepsilon_{1} W_{1}^{2}(\mathbf{e}, \kappa)+$ $\Delta_{\mathcal{F}}(|(u, v, w, d)|) \leq-\widetilde{\varrho} \bar{U}(\psi)+\Delta_{\mathcal{F}}(|(u, v, w, d)|)$, where $\widetilde{\varrho} \in\left(0, \varepsilon_{1} \min \left\{1, \gamma_{1} \rho_{1}^{*}\right\}\right)$. The same reasoning applies when $q=2$.

\footnotetext{
$\overline{{ }^{2} \text { For } x, v} \in \mathbb{R}^{n}$ and locally Lipschitz $U: \mathbb{R}^{n} \rightarrow \mathbb{R}$, let $U^{\circ}(x ; v)$ be the Clarke derivative of the function $U$ at $x$ in the direction $v$, i.e. $U^{\circ}(x ; v):=\lim \sup _{y \rightarrow x, \lambda \downarrow 0} \frac{U(y+\lambda v)-U(y)}{\lambda}$.
} 
We next investigate (P3) and distinguish two cases: transmission jumps and segment switching jumps. We only consider the case when $q=1$ as the same conclusion can be obtained for $q=2$ by following the same lines.

Case I:Transmission jumps. Let $\left(\tau_{1}, \tau_{2}, q\right) \in D_{1}^{\text {trans }}$. From Assumption 2, $\phi_{1}(0)=1 / \rho_{1}^{*}, U(\mathcal{G}(\psi))=$ $V(\xi)+\gamma_{1} \phi_{1}(0) \vartheta_{1}\left(\tau_{2}\right) W_{1}^{2}\left(h_{\mathbf{e}, 1}\left(\kappa, e_{p}, \mathbf{e}\right), \kappa+1\right) \leq$ $V(\xi)+\gamma_{1} \frac{1}{\rho_{1}^{*}} \vartheta_{1}\left(\tau_{2}\right)\left(\rho_{1} W_{1}(\mathbf{e}, \kappa)+\sigma_{1}^{v}(|v|)\right)^{2}$. In view of (A.1), $2 \rho_{1} W_{1}(\mathbf{e}, \kappa) \sigma_{1}^{v}(|v|) \leq \eta_{1} \rho_{1}^{2} W_{1}^{2}(\mathbf{e}, \kappa)+\frac{1}{\eta_{1}} \sigma_{1}^{v}(|v|)^{2}$ and recall $\left(1+\eta_{1}\right) \rho_{1}^{2}<\left(\rho_{1}^{*}\right)^{2}$ from Lemma 2 , we have that $U(\mathcal{G}(\psi)) \leq V(\xi)+\gamma_{1} \frac{1}{\rho_{1}^{*}} \vartheta_{1}\left(\tau_{2}\right)\left(1+\eta_{1}\right) \rho_{1}^{2} W_{1}^{2}(\mathbf{e}, \kappa)+$ $\gamma_{1} \frac{1}{\rho_{1}^{*}} \vartheta_{1}\left(\tau_{2}\right)\left(1+\frac{1}{\eta_{1}}\right) \sigma_{1}^{v}(|v|)^{2} \leq V(\xi)+\gamma_{1} \rho_{1}^{*} \vartheta_{1}\left(\tau_{2}\right)$ $\cdot W_{1}^{2}(\mathbf{e}, \kappa)+\Delta_{\mathcal{G}}(|v|) \leq U(\psi)+\Delta_{\mathcal{G}}(|v|)$, where $\Delta_{\mathcal{G}}(s):=$ $\frac{\gamma_{1} \lambda_{1}}{\rho_{1}^{*}}\left(1+\frac{1}{\eta_{1}}\right) \sigma_{1}^{v}(s)^{2}$.

Case II: Segment switching jumps. Let $\left(\tau_{1}, \tau_{2}, q\right) \in D_{1}^{\mathrm{seg}}$. Since $W_{2}^{2}(e, \kappa) \leq \bar{\alpha}_{W_{2}}^{2}|e|^{2} \leq \bar{\alpha}_{W_{2}}^{2} / \underline{\alpha}_{W_{1}}^{2} W_{1}^{2}(\mathbf{e}, \kappa)$, $\vartheta_{2}(0)=1$ from its definition, $\phi_{2}\left(\tau_{1}\right) \leq 1 / \rho_{2}^{*}$ and $\rho_{2}<\rho_{2}^{*}$, $U(\mathcal{G}(\psi))=V(\xi)+\gamma_{2} \phi_{2}\left(\tau_{1}\right) \vartheta_{2}(0) W_{2}^{2}(\mathbf{e}, \kappa) \leq V(\xi)+$ $\gamma_{2} \frac{1}{\rho_{2}} \frac{\bar{\alpha}_{W_{2}}^{2}}{\underline{\alpha}_{W_{1}}^{2}} W_{1}^{2}(\mathbf{e}, \kappa)=V(\xi)+\gamma_{1} \rho_{1} \frac{\gamma_{2} \bar{\alpha}_{W_{2}}^{2}}{\gamma_{1} \rho_{1} \rho_{2} \underline{\alpha}_{W_{1}}^{2}} W_{1}^{2}(\mathbf{e}, \kappa)$. Since $\tau_{2}=T_{1}$ in Case II and in view of the definition of $\bar{\lambda}_{1}, U(\psi)=V(\xi)+\gamma_{1} \phi_{1}\left(\tau_{1}\right) \lambda_{1} W_{1}^{2}(\mathbf{e}, \kappa)$. Thus, $U(\mathcal{G}(\psi)) \leq U(\psi)$, as $\phi_{1}\left(\tau_{1}\right)>\rho_{1}$ from Lemma 2 . Hence, item (P3) holds.

Let $(\psi, u, v, w, d)$ be a solution (12). Note that $U$ is locally Lipschitz in $\xi, x_{p}, \mathbf{e}, \tau_{1}, \tau_{2}$ from item (P1). In view of page 99 in (Teel \& Praly 2000), for all $i \in \mathbb{Z}_{\geq 0}$ and almost all $s \in\left[t_{i}, t_{i+1}\right], \frac{d}{d s} U(\psi(s, i)) \leq$ $U^{\circ}(\psi(s, i) ; \mathcal{F}(\psi(s, i), u(s, i), v(s, i), w(s, i), d(s, i)))$ $\leq-\widetilde{\varrho} U(\psi(s, i))+\Delta_{\mathcal{F}}(|(u(s, i), v(s, i), w(s, i), d(s, i))|)$. We then invoke the standard comparison principle to obtain $U\left(\psi\left(t_{1}, 0\right)\right) \leq \exp \left(-\widetilde{\varrho} t_{1}\right) U(\psi(0,0))+$ $\widetilde{\varrho}^{-1} \Delta_{\mathcal{F}}\left(\|(u, v, w, d)\|_{\left(t_{1}, 0\right)}\right)$. Noting that $u, v$ and $w$ do not undergo jumps, we have that $\|(u, v, w, d)\|_{\left(t_{j}, j+1\right)}=$ $\|(u, v, w, d)\|_{\left(t_{j}, j\right)}$ for any $\left(t_{j}, j\right)$ and $\left(t_{j}, j+1\right) \in$ dom $\psi$. According to item (P3), $U\left(\psi\left(t_{1}, 1\right)\right) \leq$ $U\left(\psi\left(t_{1}, 0\right)\right)+\Delta_{\mathcal{G}}\left(\|v\|_{\left(t_{1}, 1\right)}\right) \leq \exp \left(-\widetilde{\varrho} t_{1}\right) U(\psi(0,0))+$ $\widetilde{\varrho}^{-1} \Delta_{\mathcal{F}}\left(\|(u, v, w, d)\|_{\left(t_{1}, 1\right)}\right)+\Delta_{\mathcal{G}}\left(\|v\|_{\left(t_{1}, 1\right)}\right)$ when $t_{1}$ corresponds to a transmission jump instant, and $U\left(\psi\left(t_{1}, 1\right)\right)=U\left(\psi\left(t_{1}, 0\right)\right)$ when $t_{1}$ corresponds to a segment switch.

The segment jumps generate no increase of $U$ and that $\tau_{\min }>0$ is the minimum inter-transmission time for both static and dynamic segment, the following is obtained by induction. $U(\psi(t, j)) \leq \exp (-\widetilde{\varrho} t) U(\psi(0,0))+$ $\Delta_{\mathcal{F}}\left(\|(u, v, w, d)\|_{(t, j)}\right) \sum_{k=0}^{j-1} \exp \left(-\widetilde{\varrho} \tau_{\min }\right)^{k}+\widetilde{\varrho} \Delta_{\mathcal{G}}^{v}\left(\|v\|_{t, j}\right)$ $\cdot \sum_{k=0}^{j-1} \exp \left(-\widetilde{\varrho} \tau_{\min }\right)^{k}$ for all $(t, j) \in \operatorname{dom} \psi$. Consequently, using item (P1), we get that $|\psi(t, j)|_{\mathcal{A}} \leq$ $\beta\left(|\psi(0,0)|_{\mathcal{A}}, t+j\right)+\gamma\left(\|(u, v, w, d)\|_{(t, j)}\right)$, for all $(t, j) \in$ $\operatorname{dom} \psi$, where $\beta\left(s_{1}, s_{2}\right):=\underline{\alpha}_{U}^{-1} \circ\left(\exp \left(-\widetilde{\varrho} s_{2}\right) \bar{\alpha}_{U}\left(s_{1}\right)\right)$, $\gamma(s):=\frac{1}{1-\exp \left(-\widetilde{\varrho} \tau_{\min }\right)} \underline{\alpha}_{U}^{-1} \circ\left(\Delta_{\mathcal{F}}(s)+\widetilde{\varrho} \Delta_{\mathcal{G}}(s)\right)$ for $s, s_{1}, s_{2} \geq 0$ 